\title{
Semi-Pappus configurations; combinatorial generalizations of the Pappus configuration
}

\author{
Małgorzata Prażmowska · Krzysztof Prażmowski
}

Received: 23 February 2010 / Revised: 18 July 2010 / Accepted: 18 September 2010 / Published online: 10 October 2010

(C) The Author(s) 2010. This article is published with open access at Springerlink.com

\begin{abstract}
A class of partial Steiner triple systems generalizing a reduct of the classical Pappus configuration and thus called semi-Pappus configurations is defined. Fundamental geometric properties, in particular, representations and the automorphisms of semi-Pappus configurations and of the convolutions of semi-Pappus configurations and the group $C_{2}$ are established.
\end{abstract}

Keywords Partial Steiner triple system - Pappus configuration - Veblen configuration · Convolution (of a partial Steiner triple system and a group) - Affine space - Projective space

Mathematics Subject Classification (2000) $\quad 51 \mathrm{~A} 45 \cdot 51 \mathrm{E} 20$

\section{Introduction}

Via deleting the lines in some directions from an affine space one can obtain quite interesting partial linear spaces (cf. [2,4]). In particular, in that way the classical Pappus configuration embedded in the affine plane $A G(2,3)$ is obtained. This way can be further generalized to configurations contained in $A G(n, 3)$ for $n \geq 2$. On the other hand some of these configurations can be presented in terms of elementary combinatorics of finite sets; in this representation it appears that our line-reducts can be considered also as substructures of generalized Sylvester's systems (cf. [6]) which, on the other hand, generalize some generalized quadrangles (cf. [7]). These are the structures we are interested in.

Communicated by D. Ghinelli.

M. Prażmowska $(\varangle) \cdot$ K. Prażmowski

Institute of Mathematics, University of Białystok, ul. Akademicka 2, 15-267 Białystok, Poland e-mail: malgpraz@math.uwb.edu.pl

K. Prażmowski

e-mail: krzypraz@math.uwb.edu.pl 
Actually, we consider two classes of interesting substructures of generalized Sylvester's systems. The first class consists of the structures which we call semi-Pappus configurations; in the realization of a semi-Pappus configuration $\mathcal{P}_{n}$ in the affine space $A G(n, 3)$ the lines of our configuration are the affine lines with the directions that essentially linearly depend on all the vectors of the natural basis of $G F(3)^{n} . \mathcal{P}_{n}$ is a $\left(3^{n} 2^{n-1} 3^{n-1} 2^{n-1} 3\right)$-configuration. A semiPappus configuration has also a projective realization in $P G\left(3^{n}-1,2\right): \mathcal{P}_{n}$ can be defined as the substructure characterized by the following system of conditions $\left\{x_{3 i-2}+x_{3 i-1}+x_{3 i}=\right.$ $0: i=1, \ldots, n\} \cup\left\{x_{3 i-2} x_{3 i-1}+x_{3 i-1} x_{3 i}+x_{3 i-2} x_{3 i} \neq 0: i=1, \ldots, n\right\}$. For $n=2$ we obtain simply the Pappus configuration with one parallel class deleted. It appears that the deleted affine lines can be recovered in terms of the geometry of $\mathcal{P}_{n}$, which enables us to determine the automorphism groups of semi-Pappus configurations. $\mathcal{P}_{2}$ is the generalized quadrangle of order $(2,1)$, but $\mathcal{P}_{n}$ with $n>2$ is not a generalized quadrangle.

Both semi-Pappus configurations and the structures $\mathcal{M}_{n}$ which constitute the second class investigated in the paper can be considered as defined on points of an $n$-dimensional table with the lines formed by diagonals of cubes contained in the table. In the case of a semiPappus configuration we let a diagonal to pass through other points of a table. In the case of the $\left(2 \cdot 3^{n} 2^{n} 2^{n+1} 3^{n-1} 3\right)$-configuration $\mathcal{M}_{n}$ we add new intersection points of the diagonals; these points in a natural way yield another table. To get $\mathcal{M}_{n}$ we complete this table so as a semiPappus configuration is obtained. An interesting idea to proceed inductively and consider series of so constructed tables is addressed in some future work. $\mathcal{M}_{n}$ has projective realization, but it cannot be realized in any affine space over $G F(3)$. Relatively easily we determine the automorphism group of a structure $\mathcal{M}_{n}$; in essence, its characterization reduces to the problem to characterize the class of hyperplanes of a semi-Pappus configuration. Accordingly, every hyperplane in $\mathcal{P}_{n}$ can be obtained as a 'product' (under a multiplication which is the set-theoretical counterpart of the logical 'XNOR' truth function) of some number of hyperplanes of $A G(n, 3)$.

\section{Definitions}

Since the structures considered in the paper have first appeared to us as particular substructures of generalized Sylvester's systems let us start with the definition of these systems. Let $X$ be a set and $m$ be an integer. We write $\wp_{m}(X)$ for the family of $m$-element subsets of $X$. If $a, b \subset X$ we denote by $a \div b$ the symmetric difference of $a$ and $b$. A generalized Sylvester's system (see [6]) is any structure

$$
\mathbf{H}_{k}(X)=\left\langle\wp_{2 k}(X),\left\{\{a, b, a \div b\}: a, b \in \wp_{2 k}(X),|a \cap b|=k\right\}\right\rangle,
$$

where $3 k \leq|X|$. If $|X|=3 k$ then $\mathbf{H}_{k}(X)$ is isomorphic to the structure

$$
\mathbf{H}_{k}^{*}(X)=\left\langle\wp_{k}(X),\left\{\{a, b, X \backslash(a \cup b)\}: a, b \in \wp_{k}(X), a \cap b=\emptyset\right\}\right\rangle .
$$

We denote by $\mathbf{H}_{k}(n)$ any structure $\mathbf{H}_{k}(X)$ with $|X|=n$ (and similarly $\mathbf{H}_{k}^{*}(n)$ is read). Clearly, each generalized Sylvester's system is a partial Steiner triple system (i.e. a partial linear, in other words, semilinear space, with the lines of size 3 ). In particular, $\mathbf{H}_{2}(6) \cong \mathbf{H}_{2}^{*}(6)$ is the generalized quadrangle of order $(2,2)$ (see [7]).

Let $\mathfrak{M}=\langle S, \mathcal{L}\rangle$ be a partial Steiner triple system and $\mathfrak{G}=\langle G,+, 0\rangle$ be a group. Let $\mathcal{G}$ consist of the triples $(\alpha, \beta, \gamma) \in G^{3}$ such that $\alpha+\beta+\gamma=0$. The structure

$$
\mathfrak{M} \bowtie G:=\langle S \times G,\{\{(x, \alpha),(y, \beta),(z, \gamma)\}:\{x, y, z\} \in \mathcal{L},(\alpha, \beta, \gamma) \in \mathcal{G}\}\rangle
$$


is referred to as the convolution of $\mathfrak{M}$ and $\mathfrak{G}$ (cf. [8]). Clearly, it is a partial Steiner triple system.

Now, we pass to the main constructions of the paper. Let us analyse the following two procedures:

(A) Consider the affine plane $A G(2,3)$ over $G F(3)$ and delete from it the lines in two fixed directions. Equivalently: consider the Pappus configuration and delete from it a parallel class: three pairwise nonintersecting lines. That way we arrive to a $\left(9_{2} 6_{3}\right)$-configuration.

(B) Consider a $3 \times 3$ table of 9 points; for any pair of diagonals of a quadrangle contained in this table add a new point of intersection of these diagonals. In that way we obtain a configuration with 18 lines of the size 3 each and 18 points; 9 points have degree 2 , and the remaining 9 have degree 4 each.

Procedures (A) and (B) can be combined, as diagonal points obtained via (B) can be identified with the points of a suitable reduct of the affine plane $A G(2,3)$, as described in (A). That way we obtain a $\left(18_{4} 24_{3}\right)$-configuration. This configuration is obtained as the neighborhood of a pair of noncollinear and not complementary points of the generalized Sylvester's system $\mathbf{H}_{2}$ (8) (see [6]).

Procedures (A) and (B) can be generalized to "higher dimensions". Let us begin with a slightly naive verbal presentation of the structures in question.

(C) Consider a table of the size $\underbrace{3 \times \cdots \times 3}_{n \text { times }}$. This table consists of $3^{n}$ points. One can consider these points as the points of the affine space $A G(n, 3)$, so a point gets its coordinates in $(G F(3))^{n}$.

An $n$-dimensional cube contained in the table has form $I_{1} \times I_{2} \times \cdots \times I_{n}$, where $I_{i}$ is a 2-element subset of $G F(3)$ for $i=1,2, \ldots, n$. Consequently, there are $\left(\begin{array}{l}3 \\ 2\end{array}\right)^{n}=3^{n}$ cubes. For each cube we consider all its principal diagonals and a new point which is the common point of all these diagonals. For each point of a cube there is exactly one vertex opposite to it and thus every cube contains $2^{n-1}$ principal diagonals. Since each cube has $2^{n}$ vertices, each point of our table belongs to $2^{n}$ cubes. Consequently, the introduced new points of intersection of the diagonals have degree $2^{n-1}$ each and points of the table have degree $2^{n}$ (each point of the table is the end point of an exactly one diagonal in each of the cubes which contains this point). The obtained configuration has, finally, $2 \cdot 3^{n}$ points and $3^{n} \cdot 2^{n-1}$ lines of size 3 each.

(D) Let us start again from the table as in (C) and let us draw the principal diagonals of each of the cubes contained in the table. Each principal diagonal of a cube is completed to a 3-element line by the point "opposite" to the cube; if the cube is $I_{1} \times I_{2} \times \cdots \times I_{n}$ then each of its principal diagonals is completed by the point $\left[k_{1}, \ldots, k_{n}\right]$, where $I_{i} \cup\left\{k_{i}\right\}=\{0,1,2\}$ for $i=1, \ldots, n$. Note that if $a=\left[a_{1}, \ldots, a_{n}\right]$ and $b=\left[b_{1}, \ldots, b_{n}\right]$ are opposite vertices of the cube then $a_{i} \neq b_{i}$ for each $i$; the third point on the affine line through $a, b$ is $a+2(b-a)=: c$ and then $c_{i} \neq a_{i}, b_{i}$ for each $i$. Therefore, the lines which we have defined are affine lines of $A G(n, 3)$ as well.

Note, following this way, that a completed line is a principal diagonal of exactly 3 cubes and thus actually we have defined $\frac{3^{n} \cdot 2^{n-1}}{3}=3^{n-1} \cdot 2^{n-1}$ lines. Finally, we have constructed a $\left(3^{n} 2^{n-1} 3^{n-1} 2^{n-1} 3\right)$-configuration, which will be denoted by $\mathcal{P}_{n}$ and will be called a semiPappus configuration.

(E) Finally, let us identify the diagonal points of the configuration defined in $(C)$ with the vertices of the table considered in (D). Formally, this can be done, e.g., with the following stipulation. For each cube contained in the table let us identify the intersection point of its principal diagonals with the point opposite to this cube in another copy of the table; this other copy keeps its lines as they were defined in (D). The obtained configuration contains $3^{n} 2^{n-1}+$ 


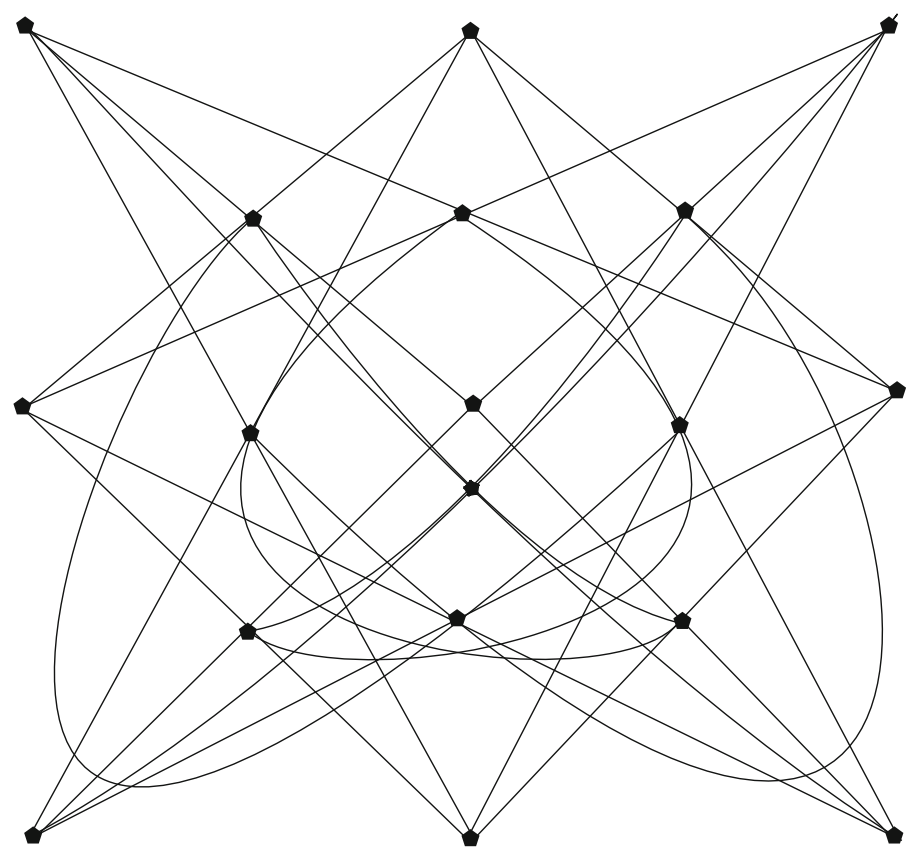

Fig. 1 The configuration $\mathcal{M}_{2}$

$3^{n-1} 2^{n-1}=2^{n-1} 3^{n-1}(3+1)$ lines. Thus we have constructed a $\left(2 \cdot 3^{n} 2^{n} 2^{n+1} 3^{n-1} 3\right)$ configuration. This configuration will be denoted by $\mathcal{M}_{n}$ (Fig. 1).

Let us present a set-theoretical representation of the configurations $\mathcal{P}_{n}$ and $\mathcal{M}_{n}$.

Representation 2.1 Consider $n$ pairwise disjoint 3-element sets $X_{1}, \ldots, X_{n}$, and a set $X_{0}$ such that $X_{0} \cap X_{i}=\emptyset$ for $i=1, \ldots, n$ and $\left|X_{0}\right|=n$. Set $Y=\bigcup_{i=1}^{n} X_{i}$ and $X=Y \cup X_{0}$. Let

$$
\mathcal{N}_{0}=\left\{a \in \wp_{n}(X):\left|a \cap X_{i}\right|=1, i=1, \ldots, n\right\} \quad \text { and } \quad \mathcal{N}=\left\{a \cup X_{0}: a \in \mathcal{N}_{0}\right\} .
$$

Then $\mathcal{N}_{0} \subset \wp_{n}(Y), \mathcal{N} \subset \wp_{2 n}(X),|Y|=3 n$, and $|X|=4 n$. Set

$$
\mathcal{N}_{2}=\left\{a \cup b: a, b \in \mathcal{N}_{0}, a \cap b=\emptyset\right\}=\left\{A \in \wp_{2 n}(X):\left|A \cap X_{i}\right|=2, i=1, \ldots, n\right\} ;
$$

then $\mathcal{N}_{2} \subset \wp_{2 n}(Y)$. Note that the boolean complementation $\varkappa$ defined on the subsets of $X$ establishes a one-to-one correspondence between the elements of $\mathcal{N}$ and $\mathcal{N}_{2}$. Similarly, the map $\tau: \mathcal{N}_{0} \ni a \longmapsto X_{0} \cup a \in \mathcal{N}$ is a bijection.

Write $X_{i}=\{(i, 0),(i, 1),(i, 2)\}$; a point $a \in \mathcal{N}_{0}$ has form $\left\{\left(1, i_{1}\right),\left(2, i_{2}\right), \ldots,\left(n, i_{n}\right)\right\}$ with $i_{1}, \ldots, i_{n} \in G F(3)$ and it can be identified with the point $\delta(a):=\left[i_{1}, \ldots, i_{n}\right]$ of the affine space $A G(n, 3)$. Under the above identification of the points in $\mathcal{N}_{0}$ and the points of $A G(n, 3)$, a cube corresponds to a set $A \in \mathcal{N}_{2}$ and the point opposite to this cube is simply $Y \backslash A \in \mathcal{N}_{0}$. The lines which are constructed via (D) have form $\{a, b, Y \backslash(a \cup b)\}$ with $a, b \in \mathcal{N}_{0}, a \cap b=\emptyset$. It is seen that $a, b \in \mathcal{N}_{0}$ are collinear in $\mathbf{H}_{n}^{*}(Y)$ iff the corresponding points of $A G(n, 3)$ are collinear in $\mathcal{P}_{n}$. It is also seen that the restriction of $\mathbf{H}_{n}^{*}(Y)$ to $\mathcal{N}_{0}$ yields a configuration isomorphic to $\mathcal{P}_{n}$. The boolean complementation defined on the subsets of $Y$ yields an isomorphism of $\mathbf{H}_{n}^{*}(Y)$ and $\mathbf{H}_{n}(Y)$ which maps $\mathcal{N}_{0}$ onto $\mathcal{N}_{2}$. Thus, finally, $\mathbf{H}_{n}(X)$, 
which contains $\mathbf{H}_{n}(Y)$, when restricted to $\mathcal{N}_{2}$ is $\mathcal{P}_{n}$. In other words: $\mathcal{P}_{n}$ is a substructure of $\mathbf{H}_{n}(3 n)$.

Via the introduced identification $\tau$, the elements of $\mathcal{N}$ can be considered as the points of $A G(n, 3)$ as well. Note that if $a, b \in \mathcal{N}_{0}$ then $a^{\prime}:=a \cup X_{0}$ and $b^{\prime}:=b \cup X_{0}$ are collinear in $\mathbf{H}_{n}(X)$ if and only if $a \cap b=\emptyset$. In that case $a^{\prime} \div b^{\prime}=a \cup b$ and the line which joins them has form $\left\{a^{\prime}, b^{\prime}, a \cup b\right\}$. This means that $a \cup b \in \mathcal{N}_{2}$ is the diagonal point of the cube $a \cup b$, as defined in accordance with (C). Therefore, the restriction of $\mathbf{H}_{n}(X)$ to $\mathcal{N}$ is the table with suitable diagonal points in $\mathcal{N}_{2}$. Finally, the restriction of $\mathbf{H}_{n}(X)$ to $\mathcal{N} \cup \mathcal{N}_{2}$ is $\mathcal{M}_{n}$, so $\mathcal{M}_{n}$ is a substructure of $\mathbf{H}_{n}(4 n)$.

The following will be also useful in 2.3. For subsets $a, b$ of $X$ we have $\varkappa(a) \div \varkappa(b)=$ $a \div b$. Consequently, in representation 2.1 the lines of $\mathcal{M}_{n}$ are the sets

$$
\{A, B, C\} \text { and }\{\varkappa(A), \varkappa(B), C\},
$$

where $\{A, B, C\}$ is a line of $\mathcal{P}_{n}$.

Note that $\mathcal{M}_{2}$ has first appeared as a neighborhood of a pair of points $a, b$ of $\mathbf{H}_{2}$ (8) with $|a \cap b|=1$. However, one can compute that there is no point in $\mathbf{H}_{3}(12)$ which is collinear with all the points in $\mathcal{P}_{3}$; thus there is no point collinear with all points in $\mathcal{M}_{3}$ as well.

Other interpretation of the structure $\mathcal{P}_{n}$ is related to some known operations on Steiner triple systems.

Representation 2.2 Let $\mathfrak{M}_{i}=\left\langle S_{i}, \mathcal{L}_{i}\right\rangle$ be a Steiner triple system for $i=1,2$. Then the standard product $\mathfrak{M}_{1} \otimes \mathfrak{M}_{2}:=\langle S, \mathcal{L}\rangle$, with $S=S_{1} \times S_{2}, \mathcal{L}=\mathcal{L}_{0}^{\prime} \cup \mathcal{L}_{1}^{\prime} \cup \mathcal{L}_{2}^{\prime}$, and

$$
\begin{array}{r}
\mathcal{L}_{0}^{\prime}:=\left\{\left\{\left(a_{1}, b_{1}\right),\left(a_{2}, b_{2}\right),\left(a_{3}, b_{3}\right)\right\}:\left\{a_{1}, a_{2}, a_{3}\right\} \in \mathcal{L}_{1},\left\{b_{1}, b_{2}, b_{3}\right\} \in \mathcal{L}_{2}\right\}, \\
\mathcal{L}_{1}^{\prime}:=\left\{\left\{\left(a_{1}, b\right),\left(a_{2}, b\right),\left(a_{3}, b\right)\right\}:\left\{a_{1}, a_{2}, a_{3}\right\} \in \mathcal{L}_{1}, b \in S_{2}\right\}, \quad \text { and } \\
\mathcal{L}_{2}^{\prime}:=\left\{\left\{\left(a, b_{1}\right),\left(a, b_{2}\right),\left(a, b_{3}\right)\right\}: a \in S_{1},\left\{b_{1}, b_{2}, b_{3}\right\} \in \mathcal{L}_{2}\right\},
\end{array}
$$

is a Steiner triple system (cf. [3]). Clearly, $\left\langle S, \mathcal{L}_{1}^{\prime} \cup \mathcal{L}_{2}^{\prime}\right\rangle$ is the Segre product of $\mathfrak{M}_{1}$ and $\mathfrak{M}_{2}$ (cf. [5,9]), and the substructure $\left\langle S, \mathcal{L}_{0}^{\prime}\right\rangle=: \mathfrak{M}_{1} \otimes \mathfrak{M}_{2}$ of $\mathfrak{M}_{1} \otimes \mathfrak{M}_{2}$ is a partial Steiner triple system. Consider the field $G F(3)$ as a single-line structure $\langle\{0,1,2\},\{\{0,1,2\}\}\rangle$; then

$$
\underbrace{(\cdots(G F(3) \widetilde{\otimes} G F(3)) \widetilde{\otimes} \cdots) \widetilde{\otimes} G F(3)}_{n \text { items }} \cong \mathcal{P}_{n} .
$$

Finally, one can give another interesting representation of the structures $\mathcal{P}_{n}$ and $\mathcal{M}_{n}$.

Representation 2.3 Consider the affine space $A G(n, 3)$ and a system of $n$ "axial" hyperplanes in it i.e. a system of $n$ hyperplanes spanned by basis axes. Let $\mathcal{L}_{0}$ consist of the lines of $A G(n, 3)$ parallel to a hyperplane in this system, then the line-reduct obtained by deleting the lines in $\mathcal{L}_{0}$ is exactly $\mathcal{P}_{n}$ (cf. similar, though less general line reducts defined in [2]). And then

$$
\mathcal{M}_{n} \cong \mathcal{P}_{n} \bowtie C_{2}
$$

is the convolution of this affine reduct and the group $C_{2}$ (comp. [8]).

\section{Geometry of the configurations $\mathcal{P}_{n}$ and $\mathcal{M}_{n}$}

There are some evident consequences of the obtained representations. Let us consider the canonical basis $\left(\mathbf{e}_{i}=[0, \ldots, 0, \underbrace{1}_{i \text {-th place }}, 0, \ldots, 0]: i=1, \ldots, n\right)$ in the vector space 
$G F(3)^{n}$, and let $\mathcal{H}_{i}$ be the hyperplane spanned by the set $\left\{\mathbf{e}_{j}: j=1, \ldots, n, j \neq i\right\}$ for $i=1, \ldots, n$. Then the lines of $\mathcal{P}_{n}$ are affine lines of $A G(n, 3)$ that are not parallel to any of the $\mathcal{H}_{i}$. Following this analytical approach we have that an affine line $a+\langle u\rangle$ is a line of $\mathcal{P}_{n}$ iff $u_{i} \neq 0$ for $i=1, \ldots, n$. Consequently, two distinct points $a, b \in G F(3)^{n}$ are collinear in $\mathcal{P}_{n}$ iff $a_{i} \neq b_{i}$ for $i=1, \ldots, n$.

Let $F(n)$ consist of the linear bijections of $G F(3)^{n}$ which leave the set $\mathcal{O}:=\bigcup\left\{\left\langle\mathbf{e}_{i}\right\rangle: i=\right.$ $1 \ldots, n\}$ invariant and let $G \mathcal{P}(n)$ be the composition

$$
G \mathcal{P}(n):=\operatorname{Tr}\left(G F(3)^{n}\right) \circ F(n)=\left\{u \mapsto \varphi(u)+b: \varphi \in F(n), b \in G F(3)^{n}\right\} .
$$

It is evident that

Fact 3.1 $G \mathcal{P}(n) \subset \operatorname{Aut}\left(\mathcal{P}_{n}\right)$ and thus each semi-Pappus configuration contains a transitive group of automorphism.

Note that $\mathcal{P}_{n}$ does not contain any Veblen subconfiguration. Actually,

Fact 3.2 A semi-Pappus configuration does not contain any triangle.

Proof Let us consider any two distinct lines $L, M$ of $\mathcal{P}_{n}$ through a point $c$; without loss of generality by 3.1 we can assume that $c=\theta$ is the zero vector. Write $L=\langle a\rangle$ and $M=\langle b\rangle$, so $L=\{\theta, a, 2 a\}$ and $M=\{\theta, b, 2 b\}$. In view of the above, $a_{i}, b_{i} \in\{1,2\}$ for each $i$. Again from 3.1, without loss of generality, we can assume now that $a=[1, \ldots, 1]$. We have $a \neq b, 2 b$. Then there is $i$ with $b_{i} \neq a_{i}$, so $b_{i}=2$. analogously, there is $j$ with $2 b_{j} \neq a_{j}$ and thus $b_{j}=1$. Since $a_{j}-b_{j}=0, a-b \| \mathcal{H}_{j}$, and since $a_{i}-2 b_{i}=0, a-2 b \| \mathcal{H}_{i}$. Thus neither the pair $a, b$ nor the pair $a, 2 b$ is collinear in $\mathcal{P}_{n}$. Analogously, neither the pair $2 a, b$ nor the pair $2 a, 2 b$ is collinear in $\mathcal{P}_{n}$.

On the other hand, a semi-Pappus configuration contains lots of quadrangles:

Fact 3.3 Let $L^{\prime}=\left\{a, b^{\prime}, c^{\prime}\right\}$ and $L^{\prime \prime}=\left\{a, b^{\prime \prime}, c^{\prime \prime}\right\}$ be two lines of $\mathcal{P}_{n}$. Then the following holds.

(i) There exists a point $d$ which completes $a, b^{\prime}, b^{\prime \prime}$ to a quadrangle in $\mathcal{P}_{n}$.

(ii) Let $d$ complete $a, b^{\prime}, b^{\prime \prime}$ to a quadrangle and let $B^{\prime \prime}=\overline{b^{\prime}, d}=\left\{b^{\prime}, d, \widetilde{b}^{\prime}\right\}, B^{\prime}=\overline{b^{\prime \prime}, d}=$ $\left\{b^{\prime \prime}, d, \widetilde{b}^{\prime \prime}\right\}$. The following conditions are equivalent:

(a) the lines $C^{\prime}=\overline{c^{\prime \prime}, \widetilde{b^{\prime}}}$ and $C^{\prime \prime}=\overline{c^{\prime}, \widetilde{b}^{\prime \prime}}$ exist in $\mathcal{P}_{n}$,

(b) $a, b^{\prime}, b^{\prime \prime}, d$ yield a parallelogram in $A G(n, 3)$.

Consequently, $C^{\prime}, C^{\prime \prime}$, if exist, cross each other.

This, together with 3.2 yields that a triple $a, b^{\prime}, b^{\prime \prime}$ has a unique completion to a quadrangle which spans in $\mathcal{P}_{n}$ a net.

Proof (i) is evident: it suffices to consider a point $d$ which completes $b^{\prime}, a, b^{\prime \prime}$ to a parallelogram in $A G(n, 3)$.

Ad (ii): To determine completions of $a, b^{\prime}, b^{\prime \prime}$ to a quadrangle, with 3.1 we assume that $a=\theta$ and then $b_{i}^{\prime}, b_{i}^{\prime \prime} \neq 0$ for $i=1, \ldots, n$. Without loss of generality, we can assume that

$$
b^{\prime}=[\underbrace{1, \ldots, 1}_{s_{1} \text { times }}, \underbrace{2, \ldots, 2}_{s_{2} \text { times }}, \underbrace{2, \ldots, 2}_{s_{3} \text { times }}] \text { and } b^{\prime \prime}=[\underbrace{1, \ldots, 1}_{s_{1} \text { times }}, \underbrace{1, \ldots, 1}_{s_{2} \text { times }}, \underbrace{2, \ldots, 2}_{s_{3} \text { times }}]
$$


with $s_{1}+s_{2}+s_{3}=n$ and $0<s_{2}<n$. Then

$$
c^{\prime}=[\underbrace{2, \ldots, 2}_{s_{1} \text { times }}, \underbrace{1, \ldots, 1}_{s_{2} \text { times }}, \underbrace{1, \ldots, 1}_{s_{3} \text { times }}] \text { and } c^{\prime \prime}=[\underbrace{2, \ldots, 2}_{s_{1} \text { times }}, \underbrace{2, \ldots, 2}_{s_{2} \text { times }}, \underbrace{1, \ldots, 1}_{s_{3} \text { times }}]
$$

Assume that $w$ completes $a, b^{\prime}, b^{\prime \prime}$ to a quadrangle; in particular, $w \neq a$. Then $w_{i} \neq b_{i}^{\prime}, b_{i}^{\prime \prime}$ for all $1 \leq i \leq n$. Thus

$$
w=[x_{1}, \ldots, x_{s_{1}}, \underbrace{0, \ldots, 0}_{s_{2} \text { times }}, z_{1}, \ldots, z_{s_{3}}]
$$

where $x_{i} \in\{0,2\}$ and $z_{j} \in\{0,1\}$ for all respective $i, j$. Direct computation gives

$$
\begin{aligned}
& \widetilde{b}^{\prime \prime}=[2+2 x_{1}, \ldots, 2+2 x_{s_{1}}, \underbrace{2, \ldots, 2}_{s_{2} \text { times }}, 1+2 z_{1}, \ldots, 1+2 z_{s_{3}}], \quad \text { and } \\
& \widetilde{b}^{\prime}=[2+2 x_{1}, \ldots, 2+2 x_{s_{1}}, \underbrace{1, \ldots, 1}_{s_{2} \text { times }}, 1+2 z_{1}, \ldots, 1+2 z_{s_{3}}] .
\end{aligned}
$$

To have $c^{\prime}, \widetilde{b}^{\prime \prime}$ collinear we need $2 x_{i} \neq 0$ for all $i$, which gives $x_{i}=2$. Analogously, $z_{j}=1$ for all $j$. Finally,

$$
w=[\underbrace{2, \ldots, 2}_{s_{1} \text { times }}, \underbrace{0, \ldots, 0}_{s_{2} \text { times }}, \underbrace{1, \ldots, 1}_{s_{3} \text { times }}]=b^{\prime}+b^{\prime \prime},
$$

as required.

Remark 3.4 For $n \geq 3$ the semi-Pappus configuration $\mathcal{P}_{n}$ is not a generalized quadrangle. It suffices to note that the point $[0,1,2, \ldots]$ is not collinear with any point on the line $\langle 1,1,1, \ldots\rangle$.

Set $I(a, b)=\left\{i: a_{i}=b_{i}\right\}$ and $\#(a, b)=|I(a, b)|$. Clearly, the number $n-\#(a, b)$ is the Hamming distance of $a, b$ in the Hamming scheme $H(n, 3)$ (see e.g. [1, Chap. 14, Sect. 3]). Analysing the proof of 3.3 we can strengthen the result as follows.

Lemma 3.5 Let $a, b^{\prime}, b^{\prime \prime}$ be as in 3.3. Then $a, b^{\prime}, b^{\prime \prime}$ has $2^{\#\left(b^{\prime}, b^{\prime \prime}\right)}-1$ completions to a quadrangle in $\mathcal{P}_{n}$. Consequently, it has a unique completion to a quadrangle iff $\#\left(b^{\prime}, b^{\prime \prime}\right)=1$.

Write $a \sim b$ when $a, b$ are distinct collinear points of $\mathcal{P}_{n}$, and $a \nsucc b$ when $a \sim b$ fails. Let $\mathcal{N}^{+}(a, b)=\{x: x \sim a, b\}$ and $\mathcal{N}^{-}(a, b)=\{x: x \neq a, b\}$. Clearly, $a=b$ iff $\#(a, b)=n, \#(a, b)=0$ iff $a \sim b$, and \# $(a, b) \neq 0$ iff $a \nsucc b$. The lemma below, though not used in the sequel, is presented here, since it gives simple and elegant direct formulas for the cardinality of the sets $\mathcal{N}^{+}(a, b)$ and $\mathcal{N}^{-}(a, b)$.

Lemma 3.6 Let \# $(a, b)=s$. Then

$$
\left|\mathcal{N}^{+}(a, b)\right|=2^{s} \text { and }\left|\mathcal{N}^{-}(a, b)\right|=3^{n}-2^{n+1}+2^{s} .
$$

Note that if $s=0$ then $\left|\mathcal{N}^{+}(a, b)\right|=1$, but then $\mathcal{N}^{+}(a, b) \cup\{a, b\}$ is a line of $\mathcal{P}_{n}$.

For fixed integer $n$ the sequence $\left(3^{n}-2^{n+1}+2^{s}: s=0, \ldots, n\right)$ is strictly increasing; consequently, we have 
Corollary 3.7 Let $a, b$ be points of $\mathcal{P}_{n}$. Then

$$
\#(a, b)=s \Longleftrightarrow\left|\mathcal{N}^{-}(a, b)\right|=3^{n}-2^{n+1}+2^{s} \Longleftrightarrow\left|\mathcal{N}^{+}(a, b)\right|=2^{s} .
$$

Consequently, for a bijection $f$ of the point set of $\mathcal{P}_{n}$ the following conditions are equivalent.

(i) $f$ is an automorphism of $\mathcal{P}_{n}$.

(ii) $f$ preserves the respective Hamming distance.

(iii) $f$ preserves (in both directions) the relation $\sim$.

Proposition 3.8 The class $\mathcal{L}_{0}$ introduced in 2.3 is definable in $\mathcal{P}_{n}$. Consequently, $A G(n, 3)$ is re-definable in $\mathcal{P}_{n}$.

Proof Let $a, b$ be a pair of distinct points noncollinear in $\mathcal{P}_{n}$. The point is to characterize the point $c$ such that $\{a, b, c\} \in \mathcal{L}_{0}$. We claim that $\{a, b, c\} \in \mathcal{L}_{0}$ iff there exists in $\mathcal{P}_{n}$ a net of the lines $\left(L_{1}\left\|L_{2}\right\| L_{3}, L_{1}^{\prime}\left\|L_{2}^{\prime}\right\| L_{3}^{\prime}\right)$ such that $a \in L_{1}, L_{1}^{\prime}, b \in L_{2}, L_{2}^{\prime}$, and $c \in L_{3}, L_{3}^{\prime}$. Indeed, if there exists such a net then, by 3.3(ii), it is a parallelogram in $A G(n, 3)$ and then $a, b, c$ are collinear in $A G(n, 3)$. Conversely, if $\{a, b, c\} \in \mathcal{L}_{0}$ then by 3.6, there exists a point $p \in \mathcal{N}^{+}(a, b)$. It suffices to complete $p, a, b$ to a parallelogram in $A G(n, 3)$; by 3.3(ii), this parallelogram yields in $\mathcal{P}_{n}$ a desired net.

As an important consequence we get

Corollary 3.9 Each automorphism of $\mathcal{P}_{n}$ is a collineation of $A G(n, 3)$.

Evidently, a linear collineation of $A G(n, 3)$ is an automorphism of $\mathcal{P}_{n}$ iff it preserves the set $\mathcal{O}$. That way we get

Proposition 3.10 $\operatorname{Aut}\left(\mathcal{P}_{n}\right)=G \mathcal{P}(n)$.

The structure $\mathbf{H}_{n}(X)$ has a projective representation in $P G(|X|-1,2)$ (cf. [6]). Consequently, $\mathcal{P}_{n}$ can be embedded into the (Fanoian) geometry $P G(3 n-1,2)$ and $\mathcal{M}_{n}$ can be embedded into $P G(4 n-1,2)$.

Proposition 3.11 Consider the embedding of $\mathcal{P}_{n}$ into $\mathbf{H}_{n}^{*}(Y)$ with $|Y|=3 n$ defined in 2.1. Let $f \in \operatorname{Aut}\left(\mathcal{P}_{n}\right)$. Then $f$ can be extended to an automorphism of $\mathbf{H}_{n}^{*}(Y)$ and, consequently, to an automorphism of $P G(3 n-1,2)$.

Proof Let $\delta$ be the bijection defined in 2.1 between $\mathcal{N}_{0} \subset \wp_{n}(Y)$ and the set of all points of $A G(n, 3)$. Let $u=\left[u_{1}, \ldots, u_{n}\right]$ be a point of $A G(n, 3)$. Each $y \in\{0,1,2\}$ yields the permutation $\gamma_{y}^{i}$ of $X_{i}$ given by $\gamma_{y}^{i}(i, v)=(i, v+y)$. Write $\gamma_{u}$ for the permutation $\bigcup\left\{\gamma_{u_{i}}^{i}: i=\right.$ $1, \ldots, n\}$ of $Y$. Take a translation $\rho_{u}, \rho_{u}: v \longmapsto v+u$ for $u, v \in G F(3)^{n}$. Let $\overline{\rho_{u}}:=\delta^{-1} \rho_{u} \delta$ be the corresponding map of $\mathcal{N}_{0}$. Then $\overline{\rho_{u}}(a)$ is the image of $a$ under $\gamma_{u}$ for each $a \in \mathcal{N}_{0}$. So, $\rho_{u}$ corresponds under $\delta$ to the permutation $\gamma_{u}$ of $Y$. Let $\varphi$ be a linear bijection of $G F(3)^{n}$ which preserves $\mathcal{O}$. Then there is a permutation $\sigma \in S_{n}$ such that $\varphi$ is defined by the formula $\varphi\left(\left[u_{1}, \ldots, u_{n}\right]\right)=\left[\alpha_{1} u_{\sigma(1)}, \ldots, \alpha_{n} u_{\sigma(n)}\right]$ with $\alpha_{i} \in\{1,2\}$. As above, we note that the map $\left[u_{1}, \ldots, u_{n}\right] \longmapsto\left[\alpha_{1} u_{1}, \ldots, \alpha_{n} u_{n}\right]$ corresponds under $\delta$ to a bijection of $Y$. Clearly, the map $\left[u_{1}, \ldots, u_{n}\right] \longmapsto\left[u_{\sigma(1)}, \ldots, u_{\sigma(n)}\right]$ corresponds to the permutation $(i, v) \longmapsto(\sigma(i), v), v \in\{0,1,2\}$. In view of 3.10 this completes the proof.

For every 3-element line $L$ the structure $L \bowtie C_{2}$ is a Veblen configuration (cf. [8]). Therefore, each line $L$ of $\mathcal{P}_{n}$ yields a Veblen subconfiguration of $\mathcal{M}_{n}=\mathcal{P}_{n} \bowtie C_{2}$ (comp 2.3). This gives, in particular, the following 
Proposition 3.12 Let $n \geq 2$. The structure $\mathcal{M}_{n}$ cannot be embedded into $A G(k, 3)$ for any integer $k$.

Proof It suffices to note that for each integer $k$, the affine space $A G(k, 3)$ does not contain any Veblen configuration.

Recall (cf. [8]) that a Veblen subconfiguration of $\mathcal{M}_{n}$ either has the form $L \bowtie C_{2}$, or it is a suitably indexed Veblen subconfiguration of $\mathcal{P}_{n}$. In view of 3.2, the class of Veblen subconfigurations of $\mathcal{M}_{n}$ is the set $\mathcal{V}=\left\{L \bowtie C_{2}: L\right.$ is a line of $\left.\mathcal{P}_{n}\right\}$.

In particular, an automorphism $f \in \operatorname{Aut}\left(\mathcal{M}_{n}\right)$ determines a permutation of the set $\mathcal{V}$ and thus $f$ determines a permutation of the lines of $\mathcal{P}_{n}$. Actually, this permutation is a collineation of $\mathcal{P}_{n}$.

Lemma 3.13 Let $\mathcal{V}_{j}=L_{j} \bowtie C_{2} \in \mathcal{V}$ with $j=1$, 2. Assume that $\mathcal{V}_{1} \neq \mathcal{V}_{2}$. The following conditions are equivalent:

(i) $\mathcal{V}_{1}, \mathcal{V}_{2}$ have a common point in $\mathcal{M}_{n}$;

(ii) the lines $L_{1}, L_{2}$ have a common point in $\mathcal{P}_{n}$.

Moreover, if $\mathcal{V}_{1}, \mathcal{V}_{2}$ have a common point $(a, i)$, then $\{(a, i),(a, 1-i)\}$ is the set of the common points of $\mathcal{V}_{1}, \mathcal{V}_{2}$ and $a$ is the common point of $L_{1}, L_{2}$.

Consequently, each $F \in \operatorname{Aut}\left(\mathcal{M}_{n}\right)$ determines a collineation $\alpha_{F} \in \operatorname{Aut}\left(\mathcal{P}_{n}\right)$ such that

$$
\begin{aligned}
F\left(L \bowtie C_{2}\right) & =\alpha_{F}(L) \bowtie C_{2} \text { and } \\
F(\{(a, 0),(a, 1)\}) & =\left\{\left(\alpha_{F}(a), 0\right),\left(\alpha_{F}(a), 1\right)\right\}
\end{aligned}
$$

for every line $L$ and every point a of $\mathcal{P}_{n}$.

Recall also that if $f \in \operatorname{Aut}\left(\mathcal{P}_{n}\right)$ then the map $f \times$ id: $(a, i) \longmapsto(f(a), i)$ where $a$ is a vector and $i \in\{0,1\}$, is an automorphism of $\mathcal{M}_{n}$.

Let us note two simple observations.

Lemma 3.14 Let $L=\{a, b, c\}$ be a line of $\mathcal{P}_{n}$ and $1 \leq i \leq n$. Then $\left\{a_{i}, b_{i}, c_{i}\right\}=\{0,1,2\}$.

Consequently, for each line $L$ and every $i=1,2, \ldots, n$ there is exactly one point $p$ on $L$ with $p_{i}=0$.

Proof It suffices to note that $L=a+\langle u\rangle$ for some vector $u$ with $u_{i} \neq 0$ for all $i$. One can write $b=a+u$ and $c=a+2 u$ and the claim is evident.

Lemma 3.15 Fix $i_{0} \in\{1, \ldots, n\}$ and define

$$
F((a, k))= \begin{cases}(a, k) & \text { if } a_{i_{0}}=0 \\ (a, 1-k) & \text { if } a_{i_{0}} \neq 0\end{cases}
$$

for every point $(a, k)$ of $\mathcal{P}_{n} \bowtie C_{2}=\mathcal{M}_{n}$. Then $F \in \operatorname{Aut}\left(\mathcal{M}_{n}\right)$. Clearly, $\alpha_{F}$ is the identity on $G F(3)^{n}$.

Proof Let $M=\left\{\left(a, k_{1}\right),\left(b, k_{2}\right),\left(c, k_{3}\right)\right\}$ be a line of $\mathcal{M}_{n}$. Then $k_{1}+k_{2}+k_{3} \equiv 0 \bmod 2$ and $L=\{a, b, c\}$ is a line of $\mathcal{P}_{n}$. In view of 3.14 one can assume that $a_{i_{0}}=0$ and $b_{i_{0}}, c_{i_{0}} \neq 0$. Then $F(M)=\left\{\left(a, k_{1}\right),\left(b, 1-k_{2}\right),\left(c, 1-k_{3}\right)\right\} ;$ since $k_{1}+\left(1-k_{2}\right)+\left(1-k_{3}\right) \equiv 0 \bmod 2, F(M)$ is a line of $\mathcal{M}_{n}$ as well. 
As an immediate consequence of 3.15 and 3.1, the group $\operatorname{Aut}\left(\mathcal{M}_{n}\right)$ is transitive.

Now, let $F \in \operatorname{Aut}\left(\mathcal{M}_{n}\right)$ be arbitrary. Consider $G=\left(\alpha_{F}{ }^{-1} \times \mathrm{id}\right) \circ F$. Then $G \in \operatorname{Aut}\left(\mathcal{M}_{n}\right)$ and $\alpha_{G}$ is the identity map. Therefore, to complete a characterization of the automorphisms of $\mathcal{M}_{n}$ it suffices to determine the kernel of the map $\alpha$ i.e. the subgroup

$$
\begin{aligned}
& \operatorname{ker}(\alpha):=\left\{F \in \operatorname{Aut}\left(\mathcal{M}_{n}\right): \alpha_{F}=\mathrm{id}\right\}=\left\{F \in \operatorname{Aut}\left(\mathcal{M}_{n}\right): F(a, k)\right. \\
& \left.\quad=(a, k) \text { or } F(a, k)=(a, 1-k), \quad \text { for all } a \in G F(3)^{n}, k \in\{0,1\}\right\} .
\end{aligned}
$$

Let $F \in \operatorname{ker}(\alpha)$, write $\mathcal{F}(F)=\{a: F(a, 0)=(a, 0)\}$; then $a \in \mathcal{F}(F)$ yields that $F(a, 1)=(a, 1)$ as well. Then $a \notin \mathcal{F}(F)$ iff $F(a, k)=(a, 1-k)$ for $k=0,1$. Generally, a given set $\mathcal{X} \subset G F(3)^{n}$ determines a map $\sigma_{\mathcal{X}}$ by the condition

$$
\sigma_{\mathcal{X}}((a, k))= \begin{cases}(a, k) & \text { when } a \in \mathcal{X} \\ (a, 1-k) & \text { when } a \notin \mathcal{X},\end{cases}
$$

(cf. (1)). Clearly, $\sigma_{\mathcal{X}}^{2}=$ id and $\mathcal{F}\left(\sigma_{\mathcal{X}}\right)=\mathcal{X}$.

Lemma 3.16 Let $\mathcal{X} \subset G F(3)^{n}$. Then $\sigma_{\mathcal{X}} \in \operatorname{Aut}\left(\mathcal{M}_{n}\right)$ iff $|L \cap \mathcal{X}| \in\{1,3\}$ for every line $L$ of $\mathcal{P}_{n}$.

Proof We can write $(a, 1-k)=(a, k+1 \bmod 2)$; clearly, $(a, k)=(a, k+0 \bmod 2)$. If $M=\left\{\left(a, k_{1}\right),\left(b, k_{2}\right),\left(c, k_{3}\right)\right\}$ is a line of $\mathcal{M}_{n}$ then $k_{1}+k_{2}+k_{3} \equiv 0 \bmod 2$. We have

$$
\sigma_{\mathcal{X}}(M)=\left\{\left(a, k_{1}+m_{1} \bmod 2\right),\left(b, k_{2}+m_{2} \bmod 2\right),\left(c, k_{3}+m_{3} \bmod 2\right)\right\},
$$

where $m_{i}=0$ if respective point is in $\mathcal{X}$ and it is 1 otherwise. The set $\sigma_{\mathcal{X}}(M)$ is a line iff $m_{1}+m_{2}+m_{3} \equiv 0 \bmod 2$ i.e. iff either no one of the $m_{i}$ is 1 or exactly two are equal 1 . This proves our claim.

Note that the conditions of 3.16 can be reformulated as follows

Corollary $3.17 \sigma_{\mathcal{X}} \in \operatorname{Aut}\left(\mathcal{M}_{n}\right)$ iff $\mathcal{X}$ is a hyperplane in $\mathcal{P}_{n}$ (i.e. iff $\mathcal{X}$ is a subspace such that each line of $\mathcal{P}_{n}$ crosses it).

For convenience, the set of all points of $\mathcal{P}_{n}$ is also considered as a hyperplane $\left(\sigma_{C_{3}^{n}}=\right.$ id $\left.\in \operatorname{Aut}\left(\mathcal{M}_{n}\right)\right)$. Let $\mathcal{H}(n)$ be the class of all hyperplanes in $\mathcal{P}_{n}$. For each $1 \leq i \leq n$ and $j \in G F(3)$ we set

$$
\lambda_{i}(j):=\left\{a \in G F(3)^{n}: a_{i}=j\right\} .
$$

Let $\Lambda(n)=\left\{\lambda_{i}(j): 1 \leq i \leq n, j \in G F(3)\right\}$. By 3.14, $\Lambda(n) \subset \mathcal{H}(n)$. For sets $\mathcal{X}^{\prime}, \mathcal{X}^{\prime \prime}$ of points we write ${ }^{1}$

$$
\mathcal{X}^{\prime} \pitchfork \mathcal{X}^{\prime \prime}:=\left(\mathcal{X}^{\prime} \cap \mathcal{X}^{\prime \prime}\right) \cup\left(C_{3}^{n} \backslash\left(\mathcal{X}^{\prime} \cup \mathcal{X}^{\prime \prime}\right)\right)
$$

Proposition 3.18 The set $\Sigma \mathcal{P}(n)=\left\{\sigma_{\mathcal{X}}: \mathcal{X} \in \mathcal{H}(n)\right\}$ is an abelian group. The group $G \mathcal{P}(n)$ acts on $\Sigma \mathcal{P}(n)$ via the formula

$$
(f \times \mathrm{id}) \circ \sigma_{\mathcal{X}}=\sigma_{f(\mathcal{X})} \circ(f \times \mathrm{id})
$$

for $f \in G \mathcal{P}(n), \mathcal{X} \in \mathcal{H}(n)$ and then

$$
\operatorname{Aut}\left(\mathcal{M}_{n}\right)=\left\{\sigma_{\mathcal{X}} \circ(f \times \mathrm{id}): f \in G \mathcal{P}(n), \mathcal{X} \in \mathcal{H}(n)\right\}
$$

1 Let $\Leftrightarrow:\{0,1\}^{2} \longrightarrow\{0,1\}$ be the boolean function which corresponds to the logical equivalence, $u \Leftrightarrow v=$ $1 \Longleftrightarrow(u=0=v) \vee(u=1=v)$ for $u, v \in\{0,1\}$. Let $\chi: \wp\left(G F(3)^{n}\right) \longrightarrow\{0,1\}$ be the characteristic function. Then $\chi_{\mathcal{X}^{\prime} \pitchfork \mathcal{X}^{\prime \prime}}=\chi_{\mathcal{X}^{\prime}} \Leftrightarrow \chi_{\mathcal{X}^{\prime \prime}}$. 
is the semidirect product $G \mathcal{P}(n) \ltimes \Sigma \mathcal{P}(n)$. The map $\sigma$ is an isomorphism of the algebra $\langle\mathcal{H}(n), \pitchfork\rangle$ and the group $\Sigma \mathcal{P}(n)$.

Proof It suffices to note the following two simple observations. First, if $\mathcal{X}_{1}, \mathcal{X}_{2}$ are two hyperplanes of $\mathcal{P}_{n}$ then the set $\mathcal{X}_{1} \pitchfork \mathcal{X}_{2}$ is a hyperplane as well. Clearly, $\mathcal{X}_{1} \pitchfork \mathcal{X}_{2}=$ $\mathcal{X}_{2} \pitchfork \mathcal{X}_{1}$ and $\mathcal{F}\left(\sigma_{\mathcal{X}_{2}} \circ \sigma_{\mathcal{X}_{1}}\right)=\mathcal{X}_{2} \pitchfork \mathcal{X}_{1}$, so $\sigma_{\mathcal{X}_{2}} \circ \sigma_{\mathcal{X}_{1}}=\sigma_{\mathcal{X}_{2} \pitchfork \mathcal{X}_{1}}=\sigma_{\mathcal{X}_{1}} \circ \sigma_{\mathcal{X}_{2}}$. Secondly, $\mathcal{F}\left((f \times \mathrm{id}) \circ \sigma_{\mathcal{X}} \circ\left(f^{-1} \times \mathrm{id}\right)\right)=f(\mathcal{X})$ for $f \in G \mathcal{P}(n), \mathcal{X} \in \mathcal{H}(n)$.

However, a hyperplane in $\mathcal{P}_{n}$ need not be a hyperplane in $A G(n, 3)$; indeed, $\lambda_{1}(0) \pitchfork$ $\lambda_{2}(0) \in \mathcal{H}(n)$, though it is not an affine hyperplane. ${ }^{2}$ Note, also, that $\lambda_{i}\left(j_{1}\right) \pitchfork \lambda_{i}\left(j_{2}\right)=$ $\lambda_{i}\left(j_{3}\right)$ whenever $\left\{j_{1}, j_{2}, j_{3}\right\}=\{0,1,2\}, i \leq n$. Write $\Lambda^{0}=\left\{\lambda_{i}(j): 1 \leq i \leq n, j \in\{0,1\}\right\}$. The following is easy.

Lemma 3.19 The set $\Lambda^{0}$ generates in $\mathcal{H}(n)$ a subgroup on $4^{n}$ elements.

Proposition 3.20 The group $\Sigma \mathcal{P}(n)$ has $4^{n}$ elements. Consequently, it is generated by the set $\left\{\sigma_{\mathcal{X}}: \mathcal{X} \in \Lambda(n)\right\}$.

Proof Denote by $\odot$ the (partial) operation which assigns to a pair $a, b$ of distinct collinear points the third point on $\overline{a, b}$. Moreover, we put $a \odot a=a$. For any two sets $\mathcal{X}, \mathcal{Y}$ of points we write $\mathcal{X} \odot \mathcal{Y}=\{x \odot y: x \in \mathcal{X}, y \in \mathcal{Y}, x \odot y$ is defined $\}$.

Let $\mathcal{X}$ be an arbitrary set of points of $\mathcal{P}_{n}$; write

$$
\mathcal{X}_{j}=\mathcal{X} \cap \lambda_{n}(j), \quad j=0,1,2 .
$$

Assume that $\mathcal{X} \in \mathcal{H}(n)$, then the following equalities hold

$$
\begin{aligned}
\mathcal{X}_{2} & =\mathcal{X}_{0} \odot \mathcal{X}_{1} \\
& =\left(\lambda_{n}(0) \backslash \mathcal{X}_{0}\right) \odot\left(\lambda_{n}(1) \backslash \mathcal{X}_{1}\right), \\
\lambda_{n}(2) \backslash \mathcal{X}_{2} & =\left(\lambda_{n}(0) \backslash \mathcal{X}_{0}\right) \odot \mathcal{X}_{1} \\
& =\mathcal{X}_{0} \odot\left(\lambda_{n}(1) \backslash \mathcal{X}_{1}\right) .
\end{aligned}
$$

Write $\mathcal{D}_{n}$ for the set of triples $\left(\mathcal{X}_{0}, \mathcal{X}_{1}, \mathcal{X}_{2}\right)$ with $\mathcal{X}_{j} \subset \lambda_{n}(j), j=0,1,2$ which satisfy (3) and (4). For further convenience for $\mathcal{X}_{i}=\emptyset=X_{j}$ we adopt $\mathcal{X}_{i} \odot X_{j}=\lambda_{n}(k),\{i, j, k\}=$ $\{0,1,2\}$. Note that, conversely, if $\left(\mathcal{X}_{0}, \mathcal{X}_{1}, \mathcal{X}_{2}\right) \in \mathcal{D}_{n}$ then $\mathcal{X}=\mathcal{X}_{0} \cup \mathcal{X}_{1} \cup \mathcal{X}_{2}$ is a hyperplane of $\mathcal{P}_{n}$.

Next, for a subset $\mathcal{X}$ of the points of $\mathcal{P}_{n}$ define the sets

$$
\mathcal{X}_{j_{1}, j_{2}}=\mathcal{X} \cap \lambda_{n}\left(j_{1}\right) \cap \lambda_{n-1}\left(j_{2}\right) .
$$

One can identify each set $\mathcal{X}_{j_{1}, j_{2}}$ with a subset $\mathcal{Y}_{j_{1}, j_{2}}$ of the points of $\mathcal{P}_{n-1}$ given by the formula

$$
\mathcal{X}_{j_{1}, j_{2}}=\mathcal{Y}_{j_{1}, j_{2}} \times\left\{j_{1}\right\} .
$$

Equations 3 and 4 can be, equivalently, rewritten in the form

$$
\begin{aligned}
\mathcal{X}_{2, k} & =\mathcal{X}_{0, i} \odot \mathcal{X}_{1, j} \\
& =\left(\lambda_{n}(0) \cap \lambda_{n-1}(i) \backslash \mathcal{X}_{0, i}\right) \odot\left(\lambda_{n}(1) \cap \lambda_{n-1}(j) \backslash \mathcal{X}_{1, j}\right), \\
\lambda_{n}(2) \cap \lambda_{n-1}(k) \backslash \mathcal{X}_{2, k} & =\left(\lambda_{n}(0) \cap \lambda_{n-1}(i) \backslash \mathcal{X}_{0, i}\right) \odot \mathcal{X}_{1, j} \\
& =\mathcal{X}_{0, i} \odot\left(\lambda_{n}(1) \cap \lambda_{n-1}(j) \backslash \mathcal{X}_{1, j}\right)
\end{aligned}
$$

$\overline{2}$ Let $\mathcal{X}$ be a hyperplane in $A G(n, 3)$. Then $\mathcal{X} \in \mathcal{H}(n)$ iff $\mathcal{X} \in \Lambda(n)$. 
for all $\{i, j, k\}=\{0,1,2\}$. Thus each $\mathcal{X} \in \mathcal{H}(n)$ determines 6 decompositions into suitable subsets $\mathcal{Y}_{j_{1}, j_{2}}$; observing the Eqs. 5 and 6 we note that each of these decompositions satisfies (3) and (4) within $\mathcal{P}_{n-1}$. This observation enables us to use a recursive reasoning as follows. Note that we prove, simultaneously, two statements:

$\alpha_{n}$ : If $\left(\mathcal{X}_{0}, \mathcal{X}_{1}, \mathcal{X}_{2}\right) \in \mathcal{D}_{n}$ and $i \leq 2$ then there exists exactly one triple $\left(\mathcal{X}_{0}^{\prime}, \mathcal{X}_{1}^{\prime}, \mathcal{X}_{2}^{\prime}\right) \in \mathcal{D}_{n}$ such that $\mathcal{X}_{i}=\mathcal{X}_{i}^{\prime}$ and $\left(\mathcal{X}_{0}, \mathcal{X}_{1}, \mathcal{X}_{2}\right) \neq\left(\mathcal{X}_{0}^{\prime}, \mathcal{X}_{1}^{\prime}, \mathcal{X}_{2}^{\prime}\right)$.

$\beta_{n}:|\mathcal{H}(n)|=4^{n}$ (this is exactly the claim of the proposition).

Step 1. Consider a triple $(\mathcal{A}, \mathcal{B}, \mathcal{C}) \in \mathcal{D}_{n-1}$ of subsets of $C_{3}^{n-1}$ and set

$$
\mathcal{X}_{0,0}=\mathcal{A} \times\{0\}, \quad \mathcal{X}_{1,1}=\mathcal{B} \times\{1\}, \quad \mathcal{X}_{2,2}=\mathcal{C} \times\{2\} .
$$

Step 2. Next, let $\left(\mathcal{A}, \mathcal{C}^{\prime}, \mathcal{B}^{\prime}\right) \in \mathcal{D}_{n-1}$ be other triple; set

$$
\mathcal{X}_{1,2}=\mathcal{B}^{\prime} \times\{1\}, \quad \mathcal{X}_{2,1}=\mathcal{C}^{\prime} \times\{2\} .
$$

Step 3. Take a triple $\left(\mathcal{C}^{\prime \prime}, \mathcal{B}, \mathcal{A}^{\prime}\right) \in \mathcal{D}_{n-1}$ and put

$$
\mathcal{X}_{0,2}=\mathcal{A}^{\prime} \times\{0\}, \quad \mathcal{X}_{2,0}=\mathcal{C}^{\prime \prime} \times\{2\} .
$$

Step 4. Find $\mathcal{B}^{\prime \prime}$ such that $\left(\mathcal{B}^{\prime \prime}, \mathcal{C}^{\prime}, \mathcal{A}^{\prime}\right) \in \mathcal{D}_{n-1}$ and set

$$
\mathcal{X}_{1,0}=\mathcal{B}^{\prime \prime} \times\{1\} .
$$

Step 5. Find $\mathcal{A}^{\prime \prime}$ with $\left(\mathcal{C}^{\prime \prime}, \mathcal{A}^{\prime \prime}, \mathcal{B}^{\prime}\right) \in \mathcal{D}_{n-1}$, set

$$
\mathcal{X}_{0,1}=\mathcal{A}^{\prime \prime} \times\{0\}
$$

Step 6. Check whether $\mathcal{X}_{1,0}, \mathcal{X}_{0,1}, \mathcal{X}_{2,2}$ satisfies (5), (6), i.e. whether $\left(\mathcal{B}^{\prime \prime}, \mathcal{A}^{\prime \prime}, \mathcal{C}\right) \in \mathcal{D}_{n-1}$.

If so, a hyperplane in $\mathcal{P}_{n}$ was obtained.

Starting with $n=2$ we find "by hand" that $|\mathcal{H}(2)|=4 \cdot 4=4^{2}$ (besides, note that based on $\mathcal{D}_{1}=\{(\{0\},\{1\},\{2\})(\{0\}, \emptyset, \varnothing),(\emptyset,\{1\}, \varnothing),(\emptyset, \emptyset,\{2\})\}$ the above procedure can be applied here). This proves $\alpha_{2}$ and $\beta_{2}$.

Assume that $\alpha_{n-1}$ and $\beta_{n-1}$ are satisfied.

Analysing the above procedure we see that if $\left(\mathcal{X}_{0}, \mathcal{X}_{1}, \mathcal{X}_{2}\right) \in \mathcal{D}_{n}$ then fixing $\mathcal{X}_{i}$ (and, consequently, fixing $\left.\mathcal{X}_{i, 0}, \mathcal{X}_{i, 1}, \mathcal{X}_{i, 2}\right)$ one can find just one another distinct triple $\left(\mathcal{X}_{0}^{\prime}, \mathcal{X}_{1}^{\prime}, \mathcal{X}_{2}^{\prime}\right) \in$ $\mathcal{D}_{n}$ with $\mathcal{X}_{i}=\mathcal{X}_{i}^{\prime}$ for each $i=0,1,2$. This proves $\alpha_{n}$.

Step 1 can be accomplished in $|\mathcal{H}(n-1)|$ ways. By $\alpha_{n-1}$, steps 2 and 3 can be accomplished in 2 ways each, and steps 4 and 5 can be accomplished in at most one way each. This proves that $\mathcal{P}_{n}$ contains at most $4 \cdot|\mathcal{H}(n-1)|$ hyperplanes. By $\beta_{n-1},|\mathcal{H}(n)| \leq 4 \cdot 4^{n-1}$. Thus by $3.19, \mathcal{P}_{n}$ contains $4^{n}$ hyperplanes, i.e. $\beta_{n}$ holds.

Open Access This article is distributed under the terms of the Creative Commons Attribution Noncommercial License which permits any noncommercial use, distribution, and reproduction in any medium, provided the original author(s) and source are credited.

\section{References}

1. Godsil C.D.: Algebraic Combinatorics. Chapmann and Hall Mathematics Series, Chapman \& Hall, New York (1993).

2. Gorodowienko J., Prażmowska M., Prażmowski K.: Elementary characterizations of some classes of reducts of affine spaces. J. Geom. 89, 17-33 (2008). 
3. Griggs T.S., Murphy J., Phelan J.S.: Anti-Pasch Steiner triple systems. J. Comb. Inf. Syst. Sci. 15, 9-84 (1990).

4. Herzer A., Meuren S.: Ein Axiomensystem für partielle affine Räume. J. Geom. 50, 124-142 (1994).

5. Naumowicz A., Prażmowski K.: On Segre's product of partial linear spaces and spaces of pencils. J. Geom. 71, 128-143 (2001).

6. Owsiejczuk A., Prażmowska M.: Combinatorial generalizations of generalized quadrangles of order (2,2). Des. Codes Cryptogr. 53, 45-57 (2009).

7. Payne S.E., Thas J.A.: Finite Generalized Quadrangles. Research Notes in Mathematics, vol. 110. Pitman, Boston (1984).

8. Prażmowska M., Prażmowski K.: The convolution of a partial Steiner triple system and a group. J. Geom. 85, 90-109 (2006).

9. Tallini G.: Partial linear spaces and algebraic varieties. Symp. Math. 28, 203-217 (1986). 